\section{BIOÉTICA Y NEUROÉTICA}

\author{
Adela Cortina \\ Universidad de Valencia \\ ORCID iD: https://orcid.org/0000-0001-7385-5374 \\ adela.cortina@uv.es \\ Jesús Conill \\ Universidad de Valencia \\ ORCID iD: https://orcid.org/0000-0003-2091-4785 \\ jesus.conill@uv.es
}

Cómo citar este artículo/Citation: Cortina, A. y Conill, J. (2019). Bioética y neuroética. Arbor, 195 (792): a503. https://doi. org/10.3989/arbor.2019.792n2004

Recibido: 30 septiembre 2015. Aceptado: 28 abril 2016.

RESUMEN: La neuroética nace oficialmente a comienzos del siglo XXI, gracias al avance de las neurociencias, como ética aplicada vinculada a la bioética, pero también como neuroética autónoma. Como ética aplicada, aborda cuestiones cercanas a la bioética. Como neuroética autónoma, se enfrenta a problemas clásicos de la filosofía desde una perspectiva neurocientífica en sentido amplio. Dos cuestiones serán centrales en ella: diseñar un marco para seleccionar, interpretar e integrar los datos de las neurociencias acerca de la moralidad, y trazar el método -o métodos- adecuados para el nuevo saber. Curiosamente, en ambos casos la mayor parte de los neuroéticos dice inscribirse en posiciones naturalistas, pero realmente procede de forma no-naturalista. El artículo se propone analizar los distintos lados de la neuroética y sacar a la luz el método que realmente siguen los neuroéticos, que desmiente al naturalismo.

PALABRAS CLAVE: Neuroética; fundamentación de la moral; ética; naturalismo; neuromejoramiento; libertad; método; juicio moral.

\section{BIOETHICS AND NEUROETHICS}

Copyright: (C) 2019 CSIC. Este es un artículo de acceso abierto distribuido bajo los términos de la licencia de uso y distribución Creative Commons Reconocimiento 4.0 Internacional (CC BY 4.0).

ABSTRACT: Neuroethics officially appeared at the start of the 21st century due to the progress made by the neurosciences, as an applied ethics related to bioethics, but also as an independent discipline in its own right. As an applied ethics, it tackles issues bordering on bioethics. As independent neuroethics, it deals with established philosophical problems from a neuroscientific standpoint in the broader sense. It involves two central questions: the design of a framework in which to select, interpret and integrate data from neuroscience on morality and outlining the appropriate method or methods for this new branch of knowledge. In both cases, most neuroethicists curiously claim to take a naturalist stance, while they actually proceed in a nonnaturalist manner. The article sets out to analyse the different facets of neuroethics and to reveal the method really used by neuroethicists, which refutes naturalism.

KEYWORDS: Neuroethics; moral foundation; ethics; naturalism; neuroenhancement; free will; method; moral judgement. 


\section{LA REVOLUCIÓN DE LAS ÉTICAS APLICADAS}

En los años sesenta y setenta del siglo XX se produce la "revolución de las éticas aplicadas" en el ámbito de la filosofía moral. Con precedentes, como cualquier otro saber, las tres pioneras son la ética del desarrollo humano, la ética económica y empresarial y la bioética. A ellas se fueron sumando la ética de los medios de comunicación, la infoética, la ética de la educación y un gran número de éticas aplicadas, que arrojan hoy en día un derroche de bibliografía en cantidad y calidad, y que han dado lugar a cátedras, institutos y asociaciones incontables.

Lo interesante de las éticas aplicadas es que constituyen una nueva forma de saber, diferente de la moral de la vida cotidiana y de las teorías éticas filosóficas, por las siguientes razones al menos (Bayertz, 2003; Cortina, 2003): 1) No las elaboran solo los filósofos, sino que trabajan conjuntamente los filósofos con los expertos de cada campo. 2) No constituyen la parte aplicada de cada teoría ética, sino que abordan los problemas de cada ámbito social contando con las teorías éticas que puedan ayudar a resolverlos en cada caso. 3) Los principios éticos no se aplican deductivamente, sino que es preciso descubrirlos en la ética cívica presente en cada uno de los ámbitos. 4) El trabajo interdisciplinar no se lleva a cabo solo en los despachos académicos, sino también en los comités y comisiones, creados por instituciones políticas o de la sociedad civil. 5) Los resultados de la reflexión y la deliberación no se dan a conocer solo a través de libros y artículos académicos, sino también mediante documentos oficiales y códigos orientadores de la acción.

Una de estas éticas aplicadas es, como se ha dicho, la bioética. Aunque tenga una larga historia, que hunde sus raíces en la tradición hipocrática, el término Bio-Ethik, como disciplina académica y como rasgo de virtud y carácter, es utilizado por primera vez por Fritz Jahr, un teólogo protestante de Halle an der Saale, en un editorial de la revista Kosmos. Jahr traduce el imperativo categórico kantiano en un imperativo bioético, que dice así: "Respeta por principio a cada ser viviente como un fin en sí mismo y trátalo, de ser posible, como a un igual" (Sass, 2011, p. 20). Con ello amplía el ámbito de la obligación ética al de todos los seres vivos, aunque el mandato en realidad no sea categórico, sino que esté condicionado a que sea posible tratar con respeto a todos los seres vivos (Gracia, 2011; Mainetti, 2011).

Sin embargo, como una nueva forma de saber, oficialmente reconocida, la bioética nace en los años setenta del siglo XX. De hecho, la Encyclopedia of Bioethics, que es la obra de referencia fundacional de la disciplina, se publica en 1978. Y la bioética nace con una cierta "bilocación", porque desde el comienzo se marcan dos tendencias: la bioética médica, representada por André Hellegers y Daniel Callahan, y la bioética ecológica, abanderada por Van Rensselaer Potter, que acuñó el nombre bioética en el artículo "Bioethics: the science of survival" (1970) y en el libro Bioethics: bridge to the future (1971) (Abel i Fabre, 2001, pp. XIII-XVII; Ferrer y Santory, 2004, p. 400). Resultó ser que la tendencia que asumió el rótulo bioética e hizo fortuna con él fue la médica, la que cobró un fuerte impulso con los principios del Informe Belmont, que no solo se convirtieron en "los principios de la bioética", sino que en ocasiones se tomaron como principios de todas las éticas profesionales (Gracia, 1989/2007). Sin embargo, desde los años noventa, gracias al fenómeno de la globalización, los avances científicos y sus consecuencias se extienden mundialmente, y cada vez resulta más insuficiente una bioética que solo atienda a las relaciones interpersonales y a las organizaciones sanitarias.

Las exigencias de un "desarrollo sostenible", que hoy se plasman en los Objetivos de Desarrollo Sostenible de las Naciones Unidas, parecen reclamar ese êthos ecológico, capaz de respetar la naturaleza y asegurar el bienestar de las generaciones presentes y futuras. No es extraño que en 1988 publique Potter su libro Global Bioethics. Y parece que es este ámbito global el que hay que tener en cuenta, además del local. De ahí que quienes consideran que el nuevo siglo exige una bioética global incluyan en ella la bioética médica, la ética de las organizaciones sanitarias, la economía de la salud, la ética ecológica, la GenÉtica y las cuestiones de justicia global (Alarcos, 2005; Cortina, 2011b; Ferrer y Santory, 2004). ¿Podemos decir que la neuroética es una de las ramas de la bioética?

\section{LA NEUROÉTICA, ¿UNA RAMA DE LA BIOÉTICA?}

La neuroética nace oficialmente el año 2002, en un Congreso celebrado en San Francisco los días 13 y 14 de mayo y auspiciado por la DANA Foundation. Al parecer, es Pontius quien utiliza por vez primera el término neuroética en 1973, y más tarde Cranford (1989) y Churchland (1991) (Amor Pan, 2015, pp. 96-98; Bonete, 2010, pp. 50-52) ${ }^{1}$. A esta última corresponde también el mérito de haber publicado en $1986 \mathrm{Neu}$ rophilosophy: Toward a Unified Science of the MindBrain, un libro pionero y extremadamente complejo y también problemático. 
El nacimiento de la neuroética tiene en su base el extraordinario auge que experimentaron las neurociencias, sobre todo a partir de los noventa del siglo XX, una década que el Congreso de los Estados Unidos declaró como "Década del Cerebro". Más tarde, en 2013, Barack Obama presenta el proyecto Brain Research through Advancing Innovative Neurotechnologies (BRAIN), y la Unión Europea, The Human Brain Project (BP), coordinado por el neurocientífico Henry Makram, también en 2013.

Naturalmente, hay muchas formas de caracterizar a las neurociencias, pero una sencilla es que son ciencias experimentales que, con todas las herramientas técnicas disponibles y utilizando el método científico de observación, experimentación e hipótesis, tienden a explicar cómo funciona el cerebro.

Sin duda las neurociencias tienen una larga historia, que arranca de Hipócrates y tiene por hitos ineludibles los representados por el tratado de Thomas Willis Cerebri Anatome (1664), el artículo de John Harlow, narrando el célebre accidente sufrido por Phineas Gage, que más tarde recoge Antonio Damasio en El error de Descartes, y por supuesto las investigaciones de Ramón y Cajal (Amor Pan, 2015, pp. 23-27; Blanco, 2014). Pero desde finales del siglo XX un conjunto de avances sitúa a las neurociencias en el primer plano de la atención, sobre todo el progreso en las técnicas de neuroimagen.

Con ayuda de las técnicas de neuroimagen es posible establecer ciertas correlaciones, por ejemplo, entre la formulación de juicios morales y determinadas áreas del cerebro, sacando a la luz, aunque con grandes cautelas, las bases neuronales de las actividades humanas, entre ellas, la moral. "Con grandes cautelas" porque la lectura de imágenes cerebrales, que en algún momento pareció asegurar un enorme avance para conocer qué zonas del cerebro se activan al ponerse en marcha determinadas conductas, está mostrando enormes limitaciones. No sabemos bien qué significa correlatos y además se necesita una gran dosis de interpretación para extraer conclusiones a partir de las imágenes cerebrales (Cortina, 2011a; Cortina, 2012; Francesco, 2007; Lavazza y de Caro, 2010).

En cualquier caso, en los últimos tiempos el prefijo neuro precede al nombre de una gran cantidad de saberes, como neuroeconomía, neuropolítica, neurorreligión y un largo etcétera. Lo neuro se ha convertido en transversal, porque conocer las bases cerebrales de cualquiera de esas actividades humanas, aun con todas las dificultades, ayuda a entenderlas mejor, lo cual es un gran avance (Cortina, 2012).
Sin embargo, el hecho de que las investigaciones neurocientíficas supongan una gran cantidad de dinero ha llevado a sospechar que sea sobre todo un negocio rentable, que promete más de lo que puede ofrecer, y que se aplicará al marketing empresarial o político con fines de manipulación, además de utilizarse para la terapia, y que las neurociencias se pueden convertir incluso en una nueva ideología. Una neurociencia crítica se abre paso (Choudhury, Nagel y Slaby, 2009; Choudhury y Slaby, 2011).

Es en este contexto de fervor neurocientífico, pero a la vez de cautelas, sospechas y críticas, en el que nace la neuroética. Según algunas de las ponencias del Congreso de San Francisco, la neuroética es "el estudio de las cuestiones éticas que se plantean a raíz de las investigaciones en el cerebro y de la aplicación de los descubrimientos a la práctica médica, a las interpretaciones legales y las políticas sanitarias y sociales" (Marcus, 2002, p. III), o bien "el examen de lo correcto e incorrecto, bueno y malo, en el tratamiento del cerebro humano, en su perfeccionamiento, o en la indeseable invasión en el cerebro o en su preocupante manipulación" (Marcus, 2002, p. 5).

Ciertamente, si la neuroética no fuera nada más que esto, sería una ética aplicada y se atendría a los principios nacidos del Informe Belmont, o bien entendería que no-maleficencia, beneficencia, autonomía y justicia constituyen la lengua común con la que se deben tratar estas cuestiones (Kucewski, 1998, pp. 423-425). De hecho, recientemente se ha convertido en un éxito de ventas el libro del neurocirujano Henry Marsh Do No Harm. La traducción castellana Ante todo, no hagas daño tal vez no haga justicia a la fuerza del juramento hipocrático y sobre todo al mandato negativo "no dañarás" que, como deber de obligación perfecta, se expresa en el principio de no-maleficencia. En cualquier caso, el libro pretende exponer las dificultades de cumplir ese deber tomando decisiones sobre actuaciones en un órgano tan complejo como el cerebro. Y, ciertamente, la neuroética, así entendida, es una boyante ética aplicada. Pero existe otra acepción de la neuroética que hace de ella una disciplina preocupada por las bases y fundamento de la moral.

Aceptando una célebre sugerencia de Roskies, es posible distinguir dos ramas de la neuroética: 1) La ética de la neurociencia, que intenta desarrollar un marco ético para regular la conducta en la investigación neurocientífica y en la aplicación del conocimiento neurocientífico a los seres humanos. Es de esta de la que nos hemos ocupado. 2) La neurociencia de la ética, que se refiere al impacto del conocimiento neu- 
rocientífico en nuestra comprensión de la ética misma y se ocupa de las bases neuronales de la agencia moral (Levy, 2007, pp. 1-2; Roskies, 2007).

Esta segunda rama consistiría en un saber interdisciplinar que pretende ser ética fundamental y que puede llegar a poner en cuestión las teorías éticas que no se compaginan con las bases neuronales descubiertas (Cortina, 2011c; Levy, 2007, p. IX).

\section{3. ÉTICA DE LAS NEUROCIENCIAS: RETOS INELUDIBLES}

La ética de las neurociencias analiza la corrección o incorrección ética de las investigaciones neurocientíficas y de su aplicación utilizando el lenguaje de la no-maleficencia, la beneficencia, la autonomía y la justicia. Sin ánimo de exhaustividad, se enfrenta a problemas específicos como los de discernir si las lecturas cerebrales pueden atentar contra el derecho a la intimidad; si en los tribunales pueden admitirse pruebas tomadas de imágenes cerebrales para exculpar a los acusados, si es legítimo utilizar las nuevas técnicas en el campo de la seguridad nacional, en relación con la "guerra contra el terror" y otras semejantes, abriendo el camino a la neuroseguridad (Moreno, 2006).

En todos estos casos importa tratar con cuidado los datos obtenidos a través de las presuntas lecturas cerebrales para no estigmatizar a determinadas personas, para no violar el deber de confidencialidad utilizando los conocimientos con fines policiales, en la lucha antiterrorista, con fines laborales o eugenésicos, y para no eximir de responsabilidades a quienes sí podían obrar de otro modo. $Y$ en todos estos casos resulta indispensable no generar expectativas que no se puedan colmar, no generar esperanzas que no se basen en estudios rigurosos (Cortina, 2011a; Cortina, 2012).

También la ética de las neurociencias se encuentra con las posibilidades de precisar más adecuadamente problemas como los del estado vegetativo y la consciencia o la muerte cerebral, como puede verse en el texto de Enrique Bonete Neuroética práctica, que dedica a ello la segunda parte del libro y recoge una muy nutrida bibliografía al respecto (Bonete, 2010).

Obviamente, tanto las investigaciones como las posibles aplicaciones que hemos mencionado plantean interrogantes éticos, pero uno de ellos se ha convertido en una auténtica estrella: la legitimidad ética del neuroenhancement, es decir, de las intervenciones de mejoramiento, y no solo de terapia. Se enfrentan en este punto autores "anti-mejora" como Michael Sandel, Leon Kass o Francis Fukuyama, autores "anti-anti-mejora”, entre los que se situarían Julian Savulescu, Nicholas
Agar, Dan Brock, Nick Bostrom, David DeGrazia, Anders Sandberg o Thomas Murria, y una posición intermedia, representada muy acertadamente por Buchanan (Buchanan 2011, pp. 1-33).

Evidentemente, los debates sobre la legitimidad del mejoramiento tienen consecuencias en políticas públicas (Schlag y Gutenberg, 2013), pero también nos llevan más allá de la neuroética entendida como ética de las neurociencias hacia la neurociencia de la ética, porque hablar de "mejorar" capacidades normales obliga a plantear la cuestión de qué es lo "normal" y lo "anormal", pero también si, en el caso de que pudiera determinarse qué es lo normal, habría que considerarlo como sagrado. ¿Hay una "naturaleza humana" que marca la normalidad y resulta intocable? ¿El método para acceder a ella es el de las ciencias experimentales o es preciso contar con métodos filosóficos? ¿Se trata de una naturaleza "naturalizada" o el naturalismo resulta insuficiente para dar cuenta de ella? (Conill Sancho, 2010; Conill Sancho, 2015; Marcos, 2010; Marcos, 2015).

Sin duda estas cuestiones nos llevan más allá de una ética de la investigación y la aplicación de los avances realizados, que asume el marco de las teorías éticas ya existentes, hacia una neuroética fundamental, que trata de averiguar cuáles son las bases cerebrales de la conducta moral. La neuroética, como neurociencia de la ética, necesita una caracterización diferente porque es ética fundamental, no ética aplicada.

\section{NEUROÉTICA FUNDAMENTAL, UN SABER AUTÓNOMO}

Los problemas a los que se enfrenta la neuroética fundamental guardan una mayor semejanza con los que ha planteado tradicionalmente la filosofía, aunque ahora desde una perspectiva neurocientífica, en el amplio sentido de la palabra. Para elaborar este saber es menester contar con la filosofía, pero también con datos de las neurociencias, la genética, la economía experimental, la psicología, la antropología y la biología evolutivas (Churchland, 2011, p. 3; Suhler y Churchland, 2011, p. 33). Desde este nuevo enfoque la neuroética se ha ido enfrentando a cuestiones como las siguientes, que exponemos sin ánimo de exhaustividad (Cortina, 2012).

El camino más frecuentado para descubrir que la neuroética es un saber peculiar fue el de la dificultad que mostraban un buen número de sujetos para avalar con argumentos sus juicios morales. El sujeto formulaba un juicio inmediato, intuitivo, y cuando se le pedían 
razones se mostraba incapaz de darlas. Ante este desconcierto moral (moral dumbfounding), autores como Haidt y Hersch señalaron que nuestros juicios morales son intuitivos, ligados a la emoción, y solo más tarde tratamos de apuntalarlos con razones (Haidt, Björklund y Murphy, 2000; Haidt y Hersch, 2001).

Tomando este hilo conductor Greene y sus colaboradores se preguntan si la razón de todo ello no es que en nuestro cerebro existen unos códigos morales, acuñados a través de la evolución y ligados a las emociones en el proceso de formación del cerebro, lo cual explicaría su carácter intuitivo y emocional. Las lecturas cerebrales practicadas a sujetos a quienes se preguntaba su posición ante dilemas morales parecían avalar esta hipótesis, porque los encuestados contestaban con mayor rapidez cuando los dilemas eran personales que cuando eran impersonales, y esta comprobación parecía refrendar la fuerza de la emoción en el primero de los casos y la mayor necesidad de reflexión racional en el segundo. La hipótesis evolutiva de que durante el proceso de formación del cerebro unos códigos morales quedaron acuñados en él, cercanos a la emoción, cobró fuerza (Greene, 2012).

El papel de las emociones en la formulación de los juicios morales se había venido convirtiendo en un tópos de la neuroética, en un lugar común (Damasio, 1994; Damasio, 2000), que no hizo sino reforzarse (Haidt et al., 2000; Haidt, 2012). Trabajos como los de Jorge Moll y sus colaboradores son una fuente de progreso en este sentido (Moll, Zahn, Oliveira-Souza, Krueger y Grafman, 2005).

Pero además algunos autores creyeron posible fundamentar una ética que, por tener una base cerebral, sería universal y podría arrumbar las teorías tradicionales (Gazzaniga, 2006; Mora, 2007). La neuroética se convertía, velis nolis, en una ética fundamental. Otros autores entendieron más prudentemente que, más que una ética universal, las neurociencias en sentido amplio permiten descubrir una estructura moral innata desde la que los seres humanos pueden hablar distintos "lenguajes morales" desde las diversas culturas (Hauser, 2006; Levy, 2007). Como es obvio, las críticas a esta posición no se hicieron esperar (Blackburn, 2008; Rorty, 2006, 27 agosto) pero, en cualquier caso, la articulación entre moral como estructura y moral como contenido volvía por sus fueros (Aranguren, 1994; Zubiri, 1986).

Ciertamente, el hilo conductor que hemos mencionado nos lleva a otro tema clásico de la ética. Desde otro ángulo, los experimentos de Benjamin Libet pu- sieron otra vez sobre el tapete la secular aporía determinismo-libertad (Cortina, 2011a, pp. 177-213; Libet, 1999; Libet, Wright y Gleason, 1982). Algunos autores la abordan desde una concepción mecanicista del cerebro que les lleva a decretar que la libertad no es más que una ilusión (Wegner, Roth, Wolf Singer, Rubia); otros la enfrentan desde una concepción del cerebro tomado como un órgano con capacidad de tomar la iniciativa (Changeux, LeDoux, Evers). Deterministas duros o incompatibilistas y también blandos o compatibilistas se enfrentan a libertaristas, también compatibilistas o incompatibilistas (Cortina, 2011a). Y se abre también camino la convicción de que contamos con dos tipos de lenguaje, que han surgido en el proceso de la evolución, el lenguaje de primera persona y el de tercera persona, y que ambos son indispensables para la vida humana. "El dualismo epistémico tiene que haber surgido de un proceso de aprendizaje y haberse acreditado en la confrontación cognitiva del homo sapiens con un mundo lleno de riesgos" (Cortina, 2011a, p. 195; Habermas, 2006, p. 174; Habermas, 2009).

Y también desde las bases cerebrales se intenta responder a problemas como el de averiguar cuál es la naturaleza de la conciencia (Arana, 2015; Morgado, 2012), en qué medida puede hablarse de la identidad del yo, si se reduce la mente al cerebro, porque "somos nuestro cerebro", o bien, en el caso de que se tratara de dos entidades, qué relación existiría entre ellas.

No es de extrañar en este contexto que la ética de las virtudes de corte aristotélico vuelva por sus fueros (Casebeer, 2003). Pero más interesante es todavía que la secular contraposición entre deontologistas y teleologistas entre de nuevo en liza y, sobre todo, que desde los descubrimientos neurocientíficos se pretenda que los primeros son emotivistas y los segundos, racionalistas, cuando la acusación de racionalismo impenitente ha sido la crítica que los deontologistas han sufrido secularmente, mientras que los teleologistas eran alabados por su capacidad para atender a las emociones (Dean, 2010, p. 10; Greene, 2014). Es esta una cuestión que tiene importantes repercusiones para la forma de entender la función del derecho (Capó, Nadal, Ramos, Fernández y Cela Conde, 2006).

Y por ir dando fin a este elenco de cuestiones, importa averiguar cuál es el modo de organización política que mejor cuadraría con las bases cerebrales de la conducta (Cortina, 2011a, pp. 99-148; García-Marzá, 2012). Como también la forma en que deberíamos educar moralmente teniendo en cuenta cómo está constituido y cómo madura el cerebro, una tarea que ocupa a la neuroeducación moral y política (Codina, 
2015), y las cuestiones de justicia social que plantea la desigualdad en el desarrollo del cerebro a causa de la pobreza material y cultural (Lipina, 2014).

Se trata de problemas clásicos, pero contemplados ahora a una nueva luz, lo cual siempre es una ganancia. Por poco que sepamos de un órgano tan misterioso como el cerebro, traspasar sus umbrales e intentar conocerlo para saber más acerca de nosotros mismos es siempre un beneficio. A fin de cuentas es lo que ha hecho la filosofía en los momentos de mayor esplendor.

Pero en el fondo de todas estas cuestiones hay dos asuntos transversales que afectan muy profundamente al modo de elaborar la neuroética:

1) Qué se entiende por "moral" cuando se intenta detectar las bases cerebrales de este tipo de actividad humana y cuál es su especificidad frente a otras formas de resolver problemas. Porque se insiste en afirmar que no existe un abismo entre el hombre y otros mamíferos, que también los chimpancés o los bonobos, entre otros, tienen instintos sociales y son capaces de una cierta vida moral, pero el desafío consiste en averiguar qué estamos entendiendo por "moral".

2) A qué métodos recurre la neuroética, si son solo científicos, en cuyo caso el naturalismo está servido, o si son también filosóficos. En el segundo caso importa saber qué métodos filosóficos y cómo se articulan con los experimentales. La discusión entre naturalismo y no naturalismo sigue en pie.

\section{DE NUEVO EL DISCURSO DEL MÉTODO. EL NATU- RALISMO A DEBATE}

Algunos neuroéticos se proponen diseñar a largo plazo una propuesta de neuroética desde el diseño de una ética filosófica integradora que unifique los avances obtenidos por las neurociencias y las restantes ciencias cognitivas (Churchland, Levy). Esta ética sería la más adecuada para comprender los distintos lados de la moralidad humana, teniendo en cuenta lo que venimos aprendiendo acerca del cerebro.

En este sentido, es paradigmático el modo de proceder de Churchland. Desde el comienzo anuncia su intención de no recurrir a ninguna instancia sobrenatural ni tampoco a ningún concepto de razón "unrealistic and rarified", en clara alusión a la propuesta kantiana. Siguiendo a Hume, se trata de descubrir las raíces de la moralidad en nuestra naturaleza, empíricamente observable: en cómo somos, de qué nos cui- damos y qué nos importa. Desde esa descripción empírica de cómo nos comportamos no derivaremos un "debe" de un "es", sino que inferiremos lo que se debe hacer, teniendo en cuenta que "inferir" es mucho más amplio que "derivar" (Churchland, 2011, p. 6).

Este temor a caer en propuestas sobrenaturales o metafísicas es un denominador común de la mayor parte de los trabajos en neuroética, que lleva a optar por el naturalismo. Ya la teoría evolucionista de Darwin ofreció una explicación naturalista del origen de la moralidad en El origen del hombre (1871), que parece refrendar la descripción de Hume; la GenÉtica, por su parte, extrajo de las predisposiciones innatas a actuar en un sentido u otro consecuencias para la calificación moral; y el avance de las neurociencias ha puesto de nuevo sobre el tapete la pretensión de explicar la moralidad sin necesidad de recurrir a ninguna otra cosa que no sea la naturaleza empíricamente descrita. Triunfan las explicaciones naturalistas, en la línea de Hume y Darwin, hasta el punto de que el naturalismo se convierte en la posición ortodoxa. El mismo Habermas, sin duda más próximo a Kant que a Hume, se reclama de un "naturalismo blando" de dificil comprensión (Habermas, 2006, p. 161; Habermas, 2009). En una línea naturalista se pronuncian, entre muchos otros, autores como Haidt, Greene, Gazzaniga, Mora, Joyce, Kitcher, y de forma muy pormenorizada, Patricia S. Churchland.

Sin embargo, una cosa es lo que se dice que se pretende hacer y otra distinta lo que realmente se hace. Porque realmente resulta imposible preguntarse por el origen de la moralidad sin contar con una noción de moralidad que permita iniciar la búsqueda. Noción que no se ha extraído de los datos empíricamente observables, sino de un concepto labrado a la vez cultural y experiencialmente. Dado el escaso espacio con el que contamos, tomaremos como ejemplo de este modo de proceder el de Churchland cuando se propone averiguar cuál es el origen de los valores morales, porque resulta paradigmático.

A juicio de Churchland, los datos empíricos avalan el hecho de que las valoraciones básicas de los seres humanos son el placer y el dolor, como decía Hume, y que están ligados con el propio cuidado y bienestar, pero también con el de otros, porque el cerebro humano es social, como el de otros mamíferos. Los cerebros de los animales sociales están organizados de tal modo que sienten placer en el ejercicio de disposiciones sociales como el cuidado de los hijos, compañeros y allegados, es decir, con aquellos con los que existe un vínculo. Sustancias neuroquímicas como la 
vasopresina y la oxitocina median en el vínculo de la pareja, los padres y los hijos, y probablemente de los parientes y allegados. Otros neurotransmisores, como la serotonina y la dopamina, juegan también un papel en la vida social, y también hormonas como la testosterona (Champagne y Curley, 2005; Churchland, 2008, p. 103; Insel y Fernald, 2004). De hecho, Churchland sugiere que tal vez la oxitocina sea la base de lo que Hume llamó "sentimiento moral".

La probabilidad de que la oxitocina, la vasopresina y un espectro de receptores sean importantes en los estilos humanos de socialidad, y su comprensión neurológica, tendrían entonces amplias implicaciones para el origen y base de la moralidad humana. Nuestros cerebros estarían estructurados para defender nuestros intereses y los de parientes y amigos.

Cómo a partir de aquí surge la moralidad requiere una respuesta evolutiva. Los seres humanos tienen instintos sociales, posibilitados por los genes, y los ajustan a las prácticas de su entorno social por el sistema de recompensa. Precisamente esos instintos son la plataforma de la cooperación y el mantenimiento del orden social y proporcionan el fundamento biológico de la ética en su más amplio sentido. Proporcionan la base para el apego a los compañeros, las crías y los parientes y amigos.

La moralidad sería entonces una dimensión de la conducta social, estrechamente ligada a nuestra disposición natural a cuidar de los demás. Sería un esquema de conducta social con cuatro dimensiones, conformado por procesos neuronales entrelazados: 1) Cuidado, enraizado en el vínculo con los parientes y amigos y en el cuidado por su bienestar; 2) reconocimiento de los estados psicológicos de los otros, enraizado en los beneficios de predecir la conducta ajena ${ }^{2}$; 3) resolución de problemas en un contexto social, por ejemplo, cómo deberíamos castigar a los infractores; y 4) aprendizaje de prácticas sociales, por refuerzo positivo y negativo, por ensayo-error, por condicionamiento y por analogía (Churchland, 2011, p. 9).

En este punto la historia confluye con propuestas que tratan de explicar el "misterio del altruismo biológico" a partir de la conformación evolutiva del homo reciprocans, de ese ser que es capaz de dar siempre que de alguna manera pueda recibir (Hauser y Levy, entre otros). Y también aquí Churchland lanzará una hipótesis que es común a otros autores: "una hipótesis plausible es que el deseo de extender el respeto y la dignidad a todos los seres humanos, más o menos limitado en un tiempo a los grupos pequeños, proba- blemente se origine aquí" (Churchland, 2008, p. 103). También Levy considera que, como la evolución refuerza las disposiciones que favorecen la adaptación, cabría pensar que nos ha dotado de un sentimiento de benevolencia que en las primeras etapas de formación del cerebro se limitaba a los cercanos pero, al cambiar el contexto, se habría ido convirtiendo en benevolencia universal; aunque añade que esto es algo especulativo (Kitcher, 2014; Levy, 2007, p. 302 y p. 303). Sin embargo, en este modo de proceder hay al menos tres presupuestos que no salen a la luz.

En primer lugar, que la benevolencia local vaya a convertirse en universal no es en modo alguno un dato empírico. Esta afirmación está por el momento tan refutada por los hechos que han surgido las propuestas de mejorar a las personas con fármacos e intervenciones cerebrales, como dijimos, por entender que la educación no está teniendo éxito.

En segundo lugar, los naturalistas tratan de descubrir el origen empírico de la moralidad y de los valores morales contando ya con una concepción de moralidad, la propia de su sociedad, la de una moral postconvencional de principios. Desde ella seleccionan los datos que caminan en esa dirección. Entienden entonces que la moralidad será cosa de cuidado, cooperación, ayuda mutua. Y si los hechos muestran que la benevolencia tiene su límite en un ser humano reciprocador, dispuesto a dar siempre que pueda esperar algún retorno, desde lo que se ha llamado "la reciprocidad indirecta", sugieren -aunque especulativamente- que el paso a la solidaridad universal se dará en el futuro.

Este modo de proceder sería legítimo si se anunciara sin ambages que no se ha echado mano de ningún concepto sobrenatural, pero sí de conceptos que han ido surgiendo culturalmente en una humanidad que ha aprendido también moralmente. Conceptos como dignidad o valor incondicionado, que exigen una actitud de respeto a aquellos a los que se atribuyen, forman parte de un mundo de predicados no-naturales, sino culturales; predicados que no describen fácticamente a un ser, pero que nos son necesarios para entendernos a nosotros mismos.

Es el caso de algún trabajo en el que Churchland aborda el concepto de dignidad y asegura que es un concepto construido, como todos los conceptos, pero que no se refiere a hechos, como otros conceptos (Churchland, 2008). Y lleva razón. Pero lo que resulta incoherente entonces es afirmar que hay épocas en las que se respeta más la dignidad que en otras y que 
hay que aprender de ellas, sin aclarar qué se quiere decir con dignidad, o con libertad e igualdad, y por qué "bioculturalmente" hemos apostado por esos valores como superiores a otros. No se trata de términos sobrenaturales, pero tampoco naturales: son términos morales, pertenecen al mundo de los valores morales, que hemos ido configurando bioculturalmente, y es preciso ir concretándolos en prácticas determinadas para entender a fondo su significado, pero necesitamos contar con ellos como orientación.

En tercer lugar, la liberación de oxitocina por conductas prosociales puede producir placer, pero también lo producen otras conductas, como la venganza o la victoria. Como apunta Evers, entre otros, nuestra identidad innata, basada neuralmente de forma especie-específica, nos dispone a desarrollar tendencias evaluativas universales como el autointerés, la disociación, la simpatía selectiva, la empatía y la xenofobia. Con lo cual, nuestra identidad neuronal como personas nos hace sociales, pero también individualistas y autoproyectivos (Evers, 2015, p. 2). A pesar de que tenemos una capacidad natural de empatía (capacidad de aprehender los estados mentales de otros), simpatía (capacidad de cuidar de otros) y asistencia mutua, el ser humano puede ser descrito también como autointeresado, y xenófobo (Evers, 2015, p. 5). No es extraño teniendo en cuenta la necesidad de cohesión con los cercanos y de protección frente a los extraños que influyó poderosamente en la conformación del cerebro. Experimentamos placer al atender a nuestros sentimientos sociales, pero también a los que favorecen nuestro interés.

¿Cómo dar cuenta a partir de hechos de la emergencia de una ética universalista, como la que impregna la Declaración Universal de Derechos Humanos de 1948, que descansa en la noción de dignidad humana? ¿No será que el aprendizaje moral es biocultural y, por lo tanto, recurre a fuentes diversas, sean filosóficas, religiosas o políticas, para ir generando la propuesta de lo que querríamos y deberíamos ser?

\section{UN MARCO DE NEUROÉTICA FUNDAMENTAL}

Por todo ello tal vez convendría cambiar de estrategia en la investigación neuroética y en vez de tomar en un comienzo métodos empíricos e intentar más tarde recurrir a alguno filosófico, aprender algo de lo que propone el Kant crítico: en vez de esperar que la experiencia empirista nos enseñe sobre moral, construir nuestros conceptos y obligar a la naturaleza a responder a las preguntas que hemos planteado desde ellos para tratar de comprender los fenómenos de la experiencia.
En realidad, eso es lo que han hecho la mayor parte de los autores que trabajan en neuroética al intentar descubrir las bases neuronales de la moralidad: contar ya con un concepto de "moral" cuando van a seleccionar en la experiencia qué fenómenos coinciden con él.

Pero también es preciso marcar una diferencia clara en relación con Kant: para diseñar ese concepto contamos con la experiencia adquirida a lo largo de nuestra historia biosocial y cultural. No hay concepto alguno que los seres humanos hayamos podido construir si no es contando con ella, pero para construirlo no nos limitamos a lo aprendido de una evolución estrictamente biológica, que no existe. El marco interpretativo al que recurrimos no surge de las constataciones empíricas, sino de esa historia natural y social a lo largo de la cual hemos ido forjando nuestros conceptos: no de una razón empírica, pero tampoco de una razón pura, sino de una razón impura (Conill Sancho, 2006). Como bien dice Habermas, a lo largo de la historia no solo hemos ido aprendiendo técnicamente, sino también moralmente. Aunque ontogenéticamente cada individuo debe hacer su aprendizaje.

Por lo tanto, la neuroética, como neurociencia de la ética, debería ser una tarea conjunta de éticos y neurocientíficos, en la que se implicarían también psicólogos, economistas experimentales, antropólogos y biólogos evolucionistas, prolongando con ello esa historia de interdisciplinariedad que es la de la sabiduría humana (Suhler y Churchland, 2011, p. 33). Su tarea consistirá entonces en:

1) Preguntarse por las bases cerebrales de la conducta moral, aclarando en qué consiste la moralidad y el mundo de categorías que sirven para comprenderla.

2) Intentar descubrir el fundamento de la obligación moral. Para atribuir una conducta moral a un ser este debe contar con unas bases corporales, entre las que se encuentra la posesión de un cerebro y un sistema nervioso, articulados con el resto del cuerpo. Pero no es lo mismo "base" que "fundamento", no es lo mismo "condición necesaria" que "condición suficiente". Sin embargo, la pregunta es si contando solo con estas bases es posible responder a la pregunta por las razones de la obligación moral, responder a la pregunta "¿por qué debo?" ante determinadas exigencias morales. La pregunta por el fundamento de la obligación no es la misma que la pregunta por las bases con las que tiene que contar un ser para ser capaz de vida moral (Cortina, 2010; Cortina, 2011a). 
3) Recurrir a teorías éticas que permitan interpretar el significado de los datos neurocientíficos para la vida moral. El saber no es un conjunto de aportaciones fragmentarias, sino que precisa un marco desde el que interpretar los datos. Sin back stories resulta imposible interpretar los datos científicos (Slaby, 2011, p. 378; Young, 2011): hay una interacción entre el marco hermenéutico y lo que puede calificarse como "descubrimientos relevantes".

4) Tratar de diseñar un marco ético, coherente con los datos más relevantes de las neurociencias, preguntarse en qué aspectos estos datos obligan a replantear elementos importantes de la teoría ética, y averiguar también en qué medida la teoría ética obliga a orientar la conducta en determinados sentidos, marcando el camino a la evolución. El cerebro humano es afortunadamente plástico.

Evidentemente, estas tareas son propias de una forma de saber que se constituye como neuroética fundamental, más que como bioética aplicada.

\section{AGRADECIMIENTOS}

Este estudio se inserta en el Proyecto de Investigación Científica y Desarrollo Tecnológico FFI201676753-C2-1, financiado por el Ministerio de Economía y Competitividad (ahora Ministerio de Ciencia, Innovación y Universidades) y en las actividades del grupo de investigación de excelencia PROMETEO 2018/121 de la Generalidad Valenciana.

\section{NOTAS}

1. M. Gazzaniga publica un primer monográfico de neuroética reconocido en 2005 (Gazzaniga, 2006), Judy Illes publica el primer importante colectivo sobre el tema en 2006, y más tarde Walter

\section{BIBLIOGRAFÍA}

Abel i Fabre, F. (2001). Bioética: orígenes, presente y futuro. Madrid: Institut Borja de Bioètica / Fundación Mapfre Medicina.

Alarcos, F. J. (2005). Bioética global, Justicia y Teología moral. Bilbao: Desclée de Brouwer / Universidad Pontificia Comillas.

Amor Pan, J. R. (2015). Bioética y Neurociencias. Barcelona: Institut Borja de Bioètica / Universitat Ramon Llull.

Arana, J. (2015). La conciencia inexplicada. Madrid: Biblioteca Nueva.

Aranguren, J. L. (1994). Ética. En: Obras Completas II. Madrid: Trotta, pp. 159502.

Bayertz, K. (2003). La moral como construcción. Una autorreflexión sobre la ética aplicada. En: Cortina, A. y García-Marzá, D. (eds.). Razón pública y éticas aplicadas: los caminos de la razón práctica en una sociedad pluralista. Madrid: Tecnos, pp. 47-70.

Blackburn, S. (2008). Response to Marc Hauser's Princeton Tanner Lectures. [En línea]. Disponible en http://www.phil. cam.ac.uk/swb24/PAPERS/Hauser.pdf

Blanco, C. (2014). Historia de la neurociencia. Madrid: Biblioteca Nueva.
Glannon en 2007. Para el nacimiento de la neuroética ver también, entre otros, J. Illes, 2006.; J. M. Giménez y S. Sánchez-Migallón, 2010; E. Bonete, 2010.

Bonete, E. (2010). Neuroética práctica. Bilbao: Desclée de Brouwer.

Buchanan, A. (2011). Beyond Humanity? Oxford: Oxford University Press. https://doi.org/10.1093/acprof:o so/9780199587810.001.0001

Capó, M., Nadal, M., Ramos, C., Fernández, C. y Cela Conde, C. J. (2006). Neuroética. Derecho y neurociencia. Ludus Vitalis, XIV (25), pp. 163-176.

Casebeer, W. D. (2003). Moral cognition and its neural constituents. Nature Reviews Neuroscience, 4 (10), pp. 840847. https://doi.org/10.1038/nrn1223

Champagne, F. P. y Curley, J. P. (2005). How social experiences influence the brain. Current Opinion in Neurobiology, 15 (6), pp. 704-709. https://doi.org/10.1016/j. conb.2005.10.001

Choudhury, S., Nagel, S. K. y Slaby. J. (2009). Critical Neuroscience: Linking Neuroscience and Society through Critical Practice. BioSocieties, 4 (1), pp. 61-77. https://doi.org/10.1017/ S1745855209006437

Choudhury, S. y Slaby, J. (eds.) (2011). Critical Neuroscience: A Handbook of the Social and Cultural Con-
2. En este punto parece ser clave el papel de las neuronas espejo, aunque es un asunto que sigue en discusión (véase Jacoboni, 2009; Rizzolatti y Sinigaglia, 2006).

texts of Neuroscience. West Sussex, UK: Wiley-Blackwell. https://doi. org/10.1002/9781444343359

Churchland, P. S. (1991). Our Brains, Our selves: Reflections on Neuroethical Questions. En Roy, D. J., Wynne, B. E. y Old, R. W. (eds.), Bioscience and Society. Nueva York: Wiley \& Sons, pp. 77-96.

Churchland, P. S. (2008). Human Dignity from a Neurophilosophical Perspective. En: President's Council on Bioethics. Human Dignity and Bioethics. Essays Commissioned by the President's Council on Bioethics. Washington: U. S. Independent Agencies and Commissions, pp. 99-121.

Churchland, P. S. (2011). Braintrust. Princeton: Princeton University Press.

Codina, M. J. (2015). Neuroeducación en virtudes cordiales. Barcelona: Octaedro.

Conill Sancho, J. (2006). Ética hermenéutica. Madrid: Tecnos.

Conill Sancho, J. (2010). De la ley natural al universalismo hermenéutico. Pensamiento. Revista de Investigación e Información Filosófica, 66 (248), pp. 227-244. 
Conill Sancho, J. (2015). Naturaleza humana en perspectiva biohermenéutica. Pensamiento. Revista de Investigación e Información Filosófica, 71 (269), pp. 1249-1260. https://doi.org/10.14422/ pen.v71.i269.y2015.011

Cortina, A. (2003). El quehacer público de la ética aplicada: ética cívica transnacional. En: Cortina, A. y García-Marzá, D. (eds.). Razón pública y éticas aplicadas: los caminos de la razón práctica en una sociedad pluralista. Madrid: Tecnos, pp. 11-44.

Cortina, A. (2010). Neuroética: ¿las bases cerebrales de una ética universal con relevancia política? Isegoría, 42, pp. 129-148. https://doi.org/10.3989/isegoria.2010.i42.687

Cortina, A. (2011a). Neuroética y neuropolítica. Madrid: Tecnos.

Cortina, A. (2011b). Globalización y Bioética. En: Romeo Casabona, C. M. (dir.). Enciclopedia de Bioderecho y Bioética I. Granada: Comares, pp. 924-936.

Cortina, A. (2011c). Neuroética: ¿ética fundamental o ética aplicada? Diálogo filosófico, 80, pp. 205-224.

Cortina, A. (coord.) (2012). Guía Comares de neurofilosofía práctica. Granada: Comares.

Cranford, R. E. (1989). The Neurologist as Ethics Consultant and as a Member of the Institutional Ethics Commitee. The Neuroethicist. Neurologic Clinics, 7 (4), pp. 697-713. https://doi.org/10.1016/ S0733-8619(18)30384-0

Damasio, A. (1994). Descartes' Error: Emotion, Reason and the Human Brain. Pan Macmillan.

Damasio, A. (2000). The Feeling of What Happens: Body and Emotion in the Making of Consciousness. Harvest Books.

Dean, R. (2010). Does Neuroscience Undermine Deontological Theory? Neuroethics, 3 (1), pp. 43-60. https://doi. org/10.1007/s12152-009-9052-x

Evers, K. (2015). Can We Be Epigenetically Proactive? En Metzinger, T. y Windt, J. M. (eds.). Open MIND 13 (T). Frankfurt am Main: MIND Group, pp. 1-21.

Ferrer, J. y Santory, A. O. (2004). Hacia una bioética global. En: Alarcos, F. J. (ed.). La moral cristiana como propuesta. Madrid: San Pablo, pp. 399-430.
Francesco, M. di (2007). Neurofilosofia, naturalismo e statuto dei giudizi morali. Ethics \& Politics, 9 (2), pp. 126-143.

García-Marzá, D. (2012). Neuropolítica: una mirada crítica sobre el poder. En Cortina, A. (coord.). Guía Comares de neurofilosofía práctica. Granada: Comares, pp. 77-96.

Gazzaniga, M. S. (2006). El cerebro ético. Barcelona: Paidós.

Giménez, J. M. y Sánchez-Migallón, S. (2010). De la Neurociencia a la Neuroética. Pamplona: EUNSA.

Glannon, W. (ed.) (2007). Defining Right and Wrong in Brain Science: Essential Readings in Neuroethics, New York: Dana Press.

Gracia, D. (1989/2007). Fundamentos de bioética. Madrid: EUDEMA.

Gracia (2011). Bioética. En: Romeo Casabona, C. M. (dir.). Enciclopedia de Bioderecho y Bioética I. Granada: Comares, pp. 209-227.

Greene, J. D. (2012). Del "es" neuronal al "debe" moral: ¿cuáles son las implicaciones morales de la psicología moral neurocientífica? En: Cortina, A. (coord.). Guía Comares de neurofilosofía práctica. Granada: Comares, pp. 149-158.

Greene, J. D. (2014). Beyond Pointand-Shoot Morality: Why Cognitive (Neuro)Science Matters for Ethics. Ethics, 124, pp. 695-726. https://doi. org/10.1086/675875

Habermas, J. (2006). Entre naturalismo y religión. Barcelona: Paidós.

Habermas, J. (2009). Philosophische Texte (Band 5). Frankfurt: Suhrkamp.

Haidt, J. (2012). El perro emocional y su cola racional: un enfoque intuicionista social del juicio moral. En: Cortina, A. (coord.). Guía Comares de neurofilosofía práctica. Granada: Comares, pp. 159-215.

Haidt, J., Björklund, F. y Murphy, S. (2000). Moral Dumbfounding: When Intuitions Finds No Reason. Lund Psychological Reports, 1 (2). [En línea]. Disponible en http://theskepticalzone.com/wp/ wp-content/uploads/2018/03/haidt. bjorklund.working-paper.when-intuition-finds-no-reason.pub603.pdf

Haidt, J.y Hersh, M. A. (2001). Sexual morality: the cultures and emotions of conservatives and liberals. Journal of Applied Social
Psychology, 31 (1), pp. 191-221. https:// doi.org/10.1111/j.1559-1816.2001. tb02489.x

Hauser, M. D. (2006). Moral Minds. New York: Harper Collins Publishers.

Illes, J. (ed.). (2006). Neuroethics. Defining the Issues in Theory. Practice and Policy. Oxford: Oxford University Press.

Insel, Th. y Fernald, R. F. (2004). How the brain processes social information: Searching for the social brain. Annual Review of Neuroscience, 27, pp. 697722. https://doi.org/10.1146/annurev. neuro.27.070203.144148

Jacoboni, M. (2009). Las neuronas espejo. Barcelona: Katz.

Kitcher, Ph. (2014). Is a naturalized ethics possible? En: Waal, F. B. M. de, Smith Churchland, P., Pievani, T. y Parmigiani, E. (eds.). Evolved Morality. The Biology and Philosophy of Human Conscience. Boston: Brill, pp. 245-260. https://doi. rg/10.1163/9789004263888_010

Kucewski, M. (1998). Casuistry. En: Chadwick, R. F. (ed.). Encyclopedia of Applied Ethics. London, San Diego: Academic Press, pp. 423-432.

Lavazza, A. y Caro, M. de (2010). Not so Fast. On Some Bold Neuroscientific Claims Concerning Human Agency. Neuroethics, 3 (1), pp. 23-41. https://doi. org/10.1007/s12152-009-9053-9

Levy, N. (2007). Neuroethics. Challenges for the $21^{\text {st }}$ Century. Cambridge: Cambridge University Press. https://doi. org/10.1017/CBO9780511811890

Libet, B. (1999). Do we have free will? Journal of Consciousness Studies, 6 (8-9), pp. 47-57.

Libet, B., Wright, E. W. y Gleason, C. A. (1982). Readiness-Potentials Preceding Unrestricted 'Spontaneous' vs. Pre-Planned Voluntary Acts. Electroencephalograph and Clinical Neurophysiology, 54, pp. 322-325. https://doi. org/10.1016/0013-4694(82)90181-X

Lipina, S. J. (2014). Consideraciones neuroéticas de la pobreza infantil. En: Salles, A. y Evers, K. (coords.). La vida social del cerebro. México: Fontamara, pp. 67-102.

Mainetti, J. A. (2011). Bioética. En: Romeo Casabona, C. M. (dir.). Enciclopedia de Bioderecho y Bioética I. Granada: Comares, pp. 227-234. 
Marcos, A. (2010). Filosofía de la naturaleza humana. Eikasia. Revista de Filosofía, 6 (35), pp. 181-208.

Marcos, A. (2015). Nuevas perspectivas en el debate sobre la naturaleza humana. Pensamiento. Revista de Información e Investigación Filosófica, 71 (269), pp. 1239-1248. https://doi.org/10.14422/ pen.v71.i269.y2015.010

Marcus, S. J. (ed.). (2002). Neuroethics: Mapping the Field. Conference Proceedings. May 13-14, 2002. San Francisco, California. New York: The Dana Press.

Marsh, H. (2014). Do No Harm. Stories of Life, Death and Brain Surgery. New York: Thomas Dunne Books / St. Martin's Press.

Moll, J., Zahn, R., Oliveira-Souza, R. de, Krueger, F. y Grafman, J. (2005). The neural basis of human moral cognition. Nature Reviews Neuroscience, 6 (10), pp. 799-809. https://doi.org/10.1038/ nrn1768

Mora, F. (2007). Neurocultura. Madrid: Alianza.
Moreno, J. D. (2006). Mind Wars. Brain Research and National Defense. New York: Dana Foundation.

Morgado, I. (2012). Cómo percibimos el mundo. Barcelona: Ariel.

Pontius, A. A. (1973). Neuro-ethics of "walking" in the newborn. Perceptual and Motor Skills, 37 (1), pp. 235-245.

Rizzolatti, G. y Sinigaglia, C. (2006). Las neuronas espejo. Barcelona: Paidós.

Rorty, R. (2006, 27 agosto). Born to Be Good. The New York Times [En línea]. Disponible en https://www.nytimes.com/2006/08/27/ books/review/Rorty.t.html

Roskies, A. L. (2007). Neuroethics for the New Millenium. En: Glannon, W. (ed.). Defining Right and Wrong in Brain Science. Essential Readings in Neuroethics. New York: Danna Press, pp. 12-18.

Sass, H. M. (2011). El pensamiento bioético de Fritz Jahr 1927-1934. Aesthethica. International Journal on Subjectivity, Politics and the Arts. Revista Internacional sobre Subjetividad, Política y Arte, 6 (2), pp. 20-33.
Schlag, S. y Gutenberg, J. (2013). Speculation and Justification in Policy-Making on Neuroenhancement. Recerca. Revista de Pensament i Anàlisi, 13, pp. 11-28. https://doi.org/10.6035/Recerca.2013.13.2

Slaby, J. (2011). Perspectiven einer kritischen Philosophie der Neurowissenschaften. Deutsche Zeitschrift für Philosophie Zweimonatsschrift der Internationalen Philosophischen Forschung, 59 (3), pp. 375-390.

Suhler, Ch. y Churchland, P. S. (2011). The neurological basis of morality. En: Illes, J. y Sahakian, B. J. (eds.). The Oxford Handbook of Neuroethics. Oxford: Oxford University Press, pp. 33-58.

Young, A. W. y Bruce, V. (2011). Understanding person perception. British Journal of Psychology, 102 (4), pp. 959974. https://doi.org/10.1111/j.20448295.2011.02045.x

Zubiri, X. (1986). Sobre el hombre. Madrid: Alianza. 